\title{
On the Flatness of Semi-Cubically Hyponormal Weighted Shifts
}

\author{
Chunji Li
}

Department of Mathematics, Hannam University, Daejeon 306-791, Korea

e-mail : chunjili@hnu.kr, chunjili2000@yahoo.com.cn

Ji Hye AHN

Department of Mathematics, Kyungpook National University, Daegu 702-701, Ko-

rea

e-mail : hyungi486@hanmail.net

Abstract. Let $W_{\alpha}$ be a weighted shift with positive weight sequence $\alpha=\left\{\alpha_{i}\right\}_{i=0}^{\infty}$. The semi-cubical hyponormality of $W_{\alpha}$ is introduced and some flatness properties of $W_{\alpha}$ are discussed in this note. In particular, it is proved that if $\alpha_{n}=\alpha_{n+1}$ for some $n \geq 1$, then $\alpha_{n+k}=\alpha_{n}$ for all $k \geq 1$.

\section{Introduction and preliminaries}

Let $\mathcal{H}$ be a separable, infinite dimensional, complex Hilbert space and let $\mathcal{L}(\mathcal{H})$ be the algebra of all bounded linear operators on $\mathcal{H}$. An operator $T$ in $\mathcal{L}(\mathcal{H})$ is weakly $n$-hyponormal if $p(T)$ is hyponormal for any polynomial $p$ with degree less than equal to $n$. And an operator $T$ is polynomially hyponormal if $p(T)$ is hyponormal for every polynomial $p$. In particular, the weak 2-hyponormality (or weak 3-hyponormality) refered to as quadratical hyponormality (or cubical hyponormality, resp.), and has been considered in detail in [5], [6] and [8]. The flatness property makes an important role for detecting the bridges between subnormal and hyponormal operators. In [10] Stampfli proved that every subnormal weighted shift $W_{\alpha}$ with any two equal weights has a flatness property, i.e., it holds that if $\alpha_{k}=\alpha_{k+1}$ for some $k \in \mathbb{N}_{0}:=\mathbb{N} \cup\{0\}$, then $\alpha_{1}=\alpha_{2}=\cdots$. In [2], R. Curto proved that the 2-hyponormal weighted shift $W_{\alpha}$ with any two equal weights has flatness properties. And he obtained a quadratically hyponormal weighted shift $W_{\alpha}$ with first two equal weights but not the flatness property and gave a question: describe all quadratically hyponormal weighted shifts with first two equal weights, which can be applied to the detections of operator gaps (cf. [CuJ], [JuP1]). But it is still open whether there exists a cubically hyponormal weighted shift $W_{\alpha}$ with a weight sequence $\alpha$ : $\alpha_{0}=\alpha_{1}<\alpha_{2}<\cdots$. Also [1], Y. Choi proved that every polynomially

Received October 29, 2008.

2000 Mathematics Subject Classification: 47B37, 47B20.

Key words and phrases: semi-cubically hyponormal, unilateral weighted shift, flatness. 
hyponormal weighted shift $W_{\alpha}$ with any two equal weights has flatness properties.

In this note we introduce a semi-cubically hyponormal weighted shift (which will be defined below) and discuss their flatness properties. In particular, we proved that if $W_{\alpha}$ is a semi-cubically hyponormal weighted shift with $\alpha_{n}=\alpha_{n+1}$ for some $n \geq 1$, then $\alpha_{n+k}=\alpha_{n}$ for all $k \geq 1$. Hence this flatness property preserves in the case of cubical hyponormality. Some of the calculations in Section 2 were obtained through computer experiments using the software tool Mathematica [12].

\section{Construction of formulas}

For $A, B \in \mathcal{L}(\mathcal{H})$, we write $[A, B]:=A B-B A$. Let $W_{\alpha}$ be a hyponormal weighted shift with a positive weight sequence $\alpha:=\left\{\alpha_{i}\right\}_{i=0}^{\infty}$. Recall that a weighted shift $W_{\alpha}$ is cubically hyponormal if $W_{\alpha}+a W_{\alpha}^{2}+b W_{\alpha}^{3}$ is hyponormal for any $a, b \in \mathbb{C}$ $([9])$, i.e.,

$$
D(a, b):=\left[\left(W_{\alpha}+a W_{\alpha}^{2}+b W_{\alpha}^{3}\right)^{*}, W_{\alpha}+a W_{\alpha}^{2}+b W_{\alpha}^{3}\right] \geq 0 \quad \text { for any } a, b \in \mathbb{C} .
$$

Let $\left\{e_{i}\right\}_{i=0}^{\infty}$ be an orthonormal basis for $\mathcal{H}$ and let $P_{n}$ be the orthogonal projection on $\vee_{i=0}^{n}\left\{e_{i}\right\}$. For $a, b \in \mathbb{C}$ we let

$$
\begin{aligned}
D_{n}(a, b)= & P_{n}\left[\left(W_{\alpha}+a W_{\alpha}^{2}+b W_{\alpha}^{3}\right)^{*}, W_{\alpha}+a W_{\alpha}^{2}+b W_{\alpha}^{3}\right] P_{n} \\
= & {\left[\begin{array}{lllllllll}
q_{0} & r_{0} & z_{0} & 0 & & & & & \\
\overline{r_{0}} & q_{1} & r_{1} & z_{1} & 0 & & & & \\
\overline{z_{0}} & \overline{r_{1}} & q_{2} & r_{2} & z_{2} & 0 & & & \\
0 & \overline{z_{1}} & \overline{r_{2}} & q_{3} & r_{3} & z_{3} & \ddots & & \\
& \ddots & \ddots & \ddots & \ddots & \ddots & \ddots & \ddots & \\
& & \ddots & \ddots & \ddots & \ddots & \ddots & \ddots & 0 \\
& & & \ddots & \ddots & \ddots & \ddots & \ddots & z_{n-2} \\
& & & & \ddots & \ddots & \ddots & \ddots & r_{n-1} \\
& & & & & 0 & \overline{z_{n-2}} & \overline{r_{n-1}} & q_{n}
\end{array}\right], }
\end{aligned}
$$

where

$$
\begin{aligned}
& q_{n}:=\left(\alpha_{n}^{2}-\alpha_{n-1}^{2}\right)+\left(\alpha_{n}^{2} \alpha_{n+1}^{2}-\alpha_{n-2}^{2} \alpha_{n-1}^{2}\right)|a|^{2}+\left(\alpha_{n}^{2} \alpha_{n+1}^{2} \alpha_{n+2}^{2}-\alpha_{n-3}^{2} \alpha_{n-2}^{2} \alpha_{n-1}^{2}\right)|b|^{2}, \\
& r_{n}:=\alpha_{n}\left(\alpha_{n+1}^{2}-\alpha_{n-1}^{2}\right) \bar{a}+\alpha_{n}\left(\alpha_{n+1}^{2} \alpha_{n+2}^{2}-\alpha_{n-1}^{2} \alpha_{n-2}^{2}\right) a \bar{b}, \\
& z_{n}:=\alpha_{n} \alpha_{n+1}\left(\alpha_{n+2}^{2}-\alpha_{n-1}^{2}\right) \bar{b} .
\end{aligned}
$$

Then it is obvious that $W_{\alpha}$ is cubically hyponormal if and only if the pentadiagonal matrix $D_{n}(a, b) \geq 0$ for any $a, b \in \mathbb{C}$ and any $n \in \mathbb{N}$. To detect $D_{n}(a, b) \geq 0$ for any $a, b \in \mathbb{C}$ and any $n \in \mathbb{N}$, we consider $d_{n}(a, b):=\operatorname{det} D_{n}(a, b)$ and it follows from [11] 
that for $n \geq 5$,

$$
\begin{aligned}
d_{n}(a, b)= & \left(q_{n-1}-\frac{r_{n-3} r_{n-2} \overline{z_{n-3}}}{\left|r_{n-3}\right|^{2}}\right) d_{n-1}-\left(\left|r_{n-2}\right|^{2}-\frac{q_{n-2} r_{n-3} r_{n-2} \overline{z_{n-3}}}{\left|r_{n-3}\right|^{2}}\right) d_{n-2} \\
& -\left(\left|z_{n-3}\right|^{2} q_{n-2}-r_{n-3} r_{n-2} \overline{z_{n-3}}\right) d_{n-3} \\
& +\left|z_{n-4}\right|^{2}\left(\left|z_{n-3}\right|^{2}-\frac{q_{n-3} r_{n-3} r_{n-2} \overline{z_{n-3}}}{\left|r_{n-3}\right|^{2}}\right) d_{n-4} \\
& +\frac{\left|z_{n-4}\right|^{2}\left|z_{n-2}\right|^{2} r_{n-3} r_{n-2} \overline{z_{n-3}}}{\left|r_{n-3}\right|^{2}} d_{n-5} .
\end{aligned}
$$

Hence if $W_{\alpha}$ is cubically hyponormal, then $d_{n}(a, b) \geq 0$ for any $a, b \in \mathbb{C}$.

Definition 2.1. (i) A weighted shift $W_{\alpha}$ is semi-cubically hyponormal with type $I$ if $W_{\alpha}+s W_{\alpha}^{3}$ is hyponormal for any $s \in \mathbb{C}$.

(ii) A weighted shift $W_{\alpha}$ is semi-cubically hyponormal with type II if $W_{\alpha}^{2}+s W_{\alpha}^{3}$ is hyponormal for any $s \in \mathbb{C}$.

2.1. Type I. Let $\left\{e_{i}\right\}_{i=0}^{\infty}$ be an orthonormal basis for $\mathcal{H}$ and let $P_{n}$ be the orthogonal projection on $\vee_{i=0}^{n}\left\{e_{i}\right\}$. For $s \in \mathbb{C}$, we let

$$
D_{n}^{[1]}(s)=P_{n}\left[\left(W_{\alpha}+s W_{\alpha}^{3}\right)^{*}, W_{\alpha}+s W_{\alpha}^{3}\right] P_{n} .
$$

It is obvious that a quadratically hyponormal weighted shift $W_{\alpha}$ is semi-cubically hyponormal with type I if and only if the matrix $D_{n}^{[1]}(s) \geq 0$ for any $s \in \mathbb{C}$ and $n \in \mathbb{N}$. Recall that for $s \in \mathbb{C}$,

$$
\begin{aligned}
D_{n}^{[1]}(s)= & P_{n}\left[\left(W_{\alpha}+s W_{\alpha}^{3}\right)^{*}, W_{\alpha}+s W_{\alpha}^{3}\right] P_{n} \\
= & {\left[\begin{array}{llllllllll}
q_{0} & 0 & z_{0} & 0 & & & & & \\
0 & q_{1} & 0 & z_{1} & 0 & & & & \\
\bar{z}_{0} & 0 & q_{2} & 0 & z_{2} & 0 & & & \\
0 & \bar{z}_{1} & 0 & q_{3} & 0 & z_{3} & \ddots & & \\
& \ddots & \ddots & \ddots & \ddots & \ddots & \ddots & \ddots & \\
& & \ddots & \ddots & \ddots & \ddots & \ddots & \ddots & 0 \\
& & & \ddots & \ddots & \ddots & \ddots & \ddots & z_{n-2} \\
& & & & \ddots & \ddots & \ddots & \ddots & 0 \\
& & & & & 0 & \bar{z}_{n-2} & 0 & q_{n}
\end{array}\right], }
\end{aligned}
$$


where

$$
\begin{aligned}
q_{k} & :=u_{k}+|s|^{2} v_{k}, \quad\left|z_{k}\right|^{2}:=|s|^{2} w_{k}, \\
u_{k} & :=\alpha_{k}^{2}-\alpha_{k-1}^{2}, \quad v_{k}:=\alpha_{k}^{2} \alpha_{k+1}^{2} \alpha_{k+2}^{2}-\alpha_{k-3}^{2} \alpha_{k-2}^{2} \alpha_{k-1}^{2}, \\
w_{k} & :=\alpha_{k}^{2} \alpha_{k+1}^{2}\left(\alpha_{k+2}^{2}-\alpha_{k-1}^{2}\right)^{2},
\end{aligned}
$$

as usual, we set $\alpha_{-1}=\alpha_{-2}=\alpha_{-3}=0$ for our convenience. To detect $D_{n}^{[1]}(s) \geq 0$ for any $s \in \mathbb{C}$ and any $n \in \mathbb{N}$, we consider $d_{n}^{[1]}:=d_{n}^{[1]}(s):=\operatorname{det} D_{n}^{[1]}(s)$. Hence if $W_{\alpha}$ is semi-cubically hyponormal with type $\mathrm{I}$, then $d_{n}^{[1]}(s) \geq 0$ for any $s \in \mathbb{C}$ and any $n \in \mathbb{N}$. Note that

$$
d_{n}^{[1]}(t)=\sum_{i=0}^{n+1} c^{[1]}(n, i) t^{i}, \quad \text { where } t:=|s|^{2} .
$$

For brevity, we will write $c(n, i)$ for $c^{[1]}(n, i)$ in this subsection without confusion.

Lemma 2.2 ([11]). The following recursive relations hold.

$$
\begin{aligned}
& d_{0}^{[1]}=u_{0}+v_{0} t \\
& d_{1}^{[1]}=u_{0} u_{1}+\left(v_{0} u_{1}+u_{0} v_{1}\right) t+v_{0} v_{1} t^{2}, \\
& d_{n}^{[1]}=q_{n} d_{n-1}^{[1]}-\left|z_{n-2}\right|^{2} q_{n-1} d_{n-3}^{[1]}+\left|z_{n-3} z_{n-2}\right|^{2} d_{n-4}^{[1]} \quad(n \geq 2),
\end{aligned}
$$

where $d_{-1}^{[1]}=1$ and $d_{-2}^{[1]}=0$.

By direct computations, we obtain the following formulas.

Lemma 2.3. The following formulas hold.

(i) $c(0,1)=v_{0}, c(0,0)=u_{0} ; c(1,2)=v_{0} v_{1}, c(1,1)=v_{0} u_{1}+u_{0} v_{1}, c(1,0)=u_{0} u_{1}$,

(ii) $c(n, 0)=u_{0} u_{1} \cdots u_{n}$,

(iii) $c(n, 1)=u_{n} c(n-1,1)+\frac{1}{u_{n-2}}\left(v_{n} u_{n-2}-w_{n-2}\right)\left(u_{0} u_{1} \cdots u_{n-2} u_{n-1}\right) \quad(n \geq 2)$,

(iv) $c(n, n+1)=v_{n} v_{n-1} \cdots v_{1} v_{0}$,

(v) $c(n, n)=u_{n}\left(v_{n-1} \cdots v_{1} v_{0}\right)+v_{n} c(n-1, n-1)-w_{n-2} v_{n-1}\left(v_{n-3} \cdots v_{1} v_{0}\right)$,

(vi) $c(n, i)=u_{n} c(n-1, i)+v_{n} c(n-1, i-1)$

$$
\begin{gathered}
+w_{n-2}\left[w_{n-3} c(n-4, i-2)-v_{n-1} c(n-3, i-2)-u_{n-1} c(n-3, i-1)\right] \\
(0 \leq i \leq n-1) .
\end{gathered}
$$

Proof. Using formulas in Lemma 2.2 and comparing the coefficients of $d_{n}^{[1]}(n \geq 0)$, we can obtain formulas in this lemma.

We now detect some flatness properties as following.

Theorem 2.4. Let $W_{\alpha}$ be a semi-cubically hyponormal weighted shift with type I. 
Then we have the following assertions.

(i) If $\alpha_{n}=\alpha_{n+1}$ for some $n \geq 1$, then $\alpha_{n+k}=\alpha_{n}$ for all $k \geq 1$.

(ii) If $\alpha_{n}=\alpha_{n+1}=\alpha_{n+2}=\alpha_{n+3}$ for some $n \geq 2$, then $\alpha_{1}=\alpha_{2}=\alpha_{3} \cdots$.

Proof. (i) We first claim that if $\alpha_{n}=\alpha_{n+1}=\alpha_{n+2}$ for some $n \in \mathbb{N}_{0}$, then $\alpha_{n+k}=\alpha_{n}$ for all $k \geq 1$. For this proof, without loss of generality, we may assume that $n=0$, i.e., $\alpha_{0}=\alpha_{1}=\alpha_{2}=1$. Then it is sufficient to show that $\alpha_{3}=1$. To do so, we detect the positivity of $D_{n}^{[1]}(t)$ for $t \geq 0$. Note that $c(n, 0)=0=c(n, 1)$. Since $d_{n}^{[1]}(t) \geq 0(t \geq 0)$, the coefficient of $t^{2}$ which is the smallest order of $d_{n}^{[1]}(t)$ should be positive. By Lemma 2.3, we have

$$
\begin{aligned}
c(4,2) & =w_{1} w_{2} c(0,0)-w_{2} v_{3} c(1,0)+v_{4} c(3,1)-w_{2} u_{3} c(1,1)+u_{4} c(3,2) \\
& =-\alpha_{4}^{2}\left(\alpha_{3}^{2}-1\right)^{3} \geq 0,
\end{aligned}
$$

which implies that $\alpha_{3}=1$. We now prove the assertion (i). Without loss of generality, we may assume $n=1$, and $\alpha_{1}=1$, i.e., $\alpha_{1}=\alpha_{2}=1$. And then it is sufficient to show that $\alpha_{3}=1$ or $\alpha_{0}=1$. Obviously $c(n, 0)=0$. The coefficient $c(n, 1)$ of $t$ which is the smallest order of $d_{n}(t)$ is positive, but since

$$
\begin{aligned}
c(4,1) & =u_{4} c(3,1)+\frac{1}{u_{2}}\left(v_{4} u_{2}-w_{2}\right)\left(u_{0} u_{1} u_{2} u_{3}\right) \\
& =\alpha_{4}^{2} \alpha_{0}^{2}\left(1-\alpha_{3}^{2}\right)^{3}\left(1-\alpha_{0}^{2}\right),
\end{aligned}
$$

we have $\alpha_{0}=1$ or $\alpha_{3}=1$. Hence by the above claim we have this conclusion.

(ii) Without loss of generality, we may assume $n=2$, i.e., $\alpha_{2}=\alpha_{3}=\alpha_{4}=\alpha_{5}=1$. Then it is sufficient to show that $\alpha_{1}=1$. First note that $c(n, 0)=0$. By Lemma 2.3 , we have $c(3,1)=\alpha_{0}^{4}\left(\alpha_{1}^{2}-1\right)^{3} \geq 0$, and so $\alpha_{1} \geq 1$. Hence $\alpha_{1}=1$.

2.2. Type II. We use the same idea with type I. By direct calculation, we have that

$$
\begin{aligned}
D_{n}^{[2]}: & =D_{n}^{[2]}(s)=\left[\left(W_{\alpha}^{2}+s W_{\alpha}^{3}\right)^{*},\left(W_{\alpha}^{2}+s W_{\alpha}^{3}\right)\right] \\
& =\left[\begin{array}{ccccccccc}
\omega_{0} & \bar{\phi}_{0} & 0 & & & & & \\
\phi_{0} & \omega_{1} & \bar{\phi}_{1} & 0 & & & & \\
0 & \phi_{1} & \omega_{2} & \bar{\phi}_{2} & 0 & & & \\
& 0 & \phi_{2} & \omega_{3} & \bar{\phi}_{3} & 0 & & \\
& & 0 & \phi_{3} & \omega_{4} & \bar{\phi}_{4} & \ddots & \\
& & & 0 & \phi_{4} & \omega_{5} & \ddots & \ddots \\
& & & & \ddots & \ddots & \ddots & \ddots \\
& & & & & \ddots & \ddots & \ddots
\end{array}\right],
\end{aligned}
$$


where

$$
\begin{aligned}
\omega_{k} & :=\xi_{k}+|s|^{2} \eta_{k}, \\
\phi_{k} & :=s \sqrt{\delta_{k}} \\
\xi_{k} & :=\alpha_{k}^{2} \alpha_{k+1}^{2}-\alpha_{k-2}^{2} \alpha_{k-1}^{2}, \\
\eta_{k} & :=\alpha_{k}^{2} \alpha_{k+1}^{2} \alpha_{k+2}^{2}-\alpha_{k-3}^{2} \alpha_{k-2}^{2} \alpha_{k-1}^{2}, \\
\delta_{k} & :=\alpha_{k}^{2}\left(\alpha_{k+1}^{2} \alpha_{k+2}^{2}-\alpha_{k-2}^{2} \alpha_{k-1}^{2}\right)^{2} \quad(k \geq 0),
\end{aligned}
$$

and $\alpha_{-1}=\alpha_{-2}=\alpha_{-3}:=0$. Clearly, $W_{\alpha}^{2}+s W_{\alpha}^{3}$ is hyponormal if and only if $D_{n}^{[2]}(s) \geq 0$ for every $s \in \mathbb{C}$ and every $n \geq 0$. Let $d_{n}^{[2]}(\cdot):=\operatorname{det}\left(D_{n}^{[2]}(\cdot)\right)$. Then

$$
\begin{aligned}
d_{0}^{[2]} & =\omega_{0}, \\
d_{1}^{[2]} & =\omega_{0} \omega_{1}-\left|\phi_{0}\right|^{2}, \\
d_{n+2}^{[2]} & =\omega_{n+2} d_{n+1}^{[2]}-\left|\phi_{n+1}\right|^{2} d_{n}^{[2]} \quad(n \geq 0),
\end{aligned}
$$

and that $d_{n}^{[2]}$ is actually a polynomial in $t:=|s|^{2}$ of degree $n+1$, with Maclaurin expansion $d_{n}^{[2]}(t):=\sum_{i=0}^{n+1} c^{[2]}(n, i) t^{i}$. This gives at once the following lemma. For brevity, we will write $c(n, i)$ for $c^{[2]}(n, i)$ in Subsection 2.2 without confusion.

Lemma 2.5. It holds that

(i) $c(0,0)=\xi_{0}, \quad c(0,1)=\eta_{0}$,

(ii) $c(1,0)=\xi_{1} \xi_{0}, \quad c(1,1)=\xi_{1} \eta_{0}+\xi_{0} \eta_{1}, \quad c(1,2)=\eta_{1} \eta_{0}$,

(iii) $c(n, 0)=\xi_{0} \xi_{1} \cdots \xi_{n}>0$,

(iv) $c(n, n+1)=\eta_{0} \eta_{1} \cdots \eta_{n}>0$

(v) $c(n+2, i)=\xi_{n+2} c(n+1, i)+\eta_{n+2} c(n+1, i-1)-\delta_{n+1} c(n, i-1)$

$$
(n \geq 0, \quad 0 \leq i \leq n+3) .
$$

Proof. Repeat the methods which were used in Lemma 2.3.

Theorem 2.6. If $W_{\alpha}$ is semi-cubically hyponormal with type II such that $\alpha_{n}=$ $\alpha_{n+1}$ for some $n \geq 1$, then $\alpha_{n+k}=\alpha_{n}$ for all $k \geq 1$.

Proof. We first prove that if $\alpha_{n}=\alpha_{n+1}=\alpha_{n+2}$ for some $n$, then $\alpha_{n+k}=\alpha_{n}$ for all $k \geq 1$. For this proof, without loss of generality, we assume that $n=0$, i.e., $\alpha_{0}=\alpha_{1}=\alpha_{2}=1$. Then it is sufficient to show that $\alpha_{3}=1$. In fact,

$$
c(6,0)=-\alpha_{5}^{2} \alpha_{4}^{2}\left(\alpha_{3}^{2}-1\right)\left(\alpha_{3}^{2} \alpha_{4}^{2}-1\right)\left(\alpha_{4}^{2} \alpha_{5}^{2}-\alpha_{3}^{2}\right)\left(\alpha_{5}^{2} \alpha_{6}^{2}-\alpha_{3}^{2} \alpha_{4}^{2}\right),
$$

and $d_{6}^{[2]}(t) \geq 0$ for all $t \geq 0$, we must have $\alpha_{3}=1$. By using this claim, we prove this theorem. Without loss of generality, we may assume $n=1$, and $\alpha_{1}=1$, i.e., $\alpha_{1}=\alpha_{2}=1$. By the positivity of $c(6,0)$, we obtain $\alpha_{3}=\alpha_{0}=1$. Hence the above assertion about three equal weights proves this theorem. 
Theorems 2.4 and 2.6 provide a question about the flatness of semi-cubically hyponormal weighted shifts as the following problem.

Problem 2.7. Let $W_{\alpha}$ be a semi-cubically hyponormal weighted shift with a weight sequence $\alpha=\left\{\alpha_{i}\right\}_{i=0}^{\infty}$ satisfying $\alpha_{n}=\alpha_{n+1}$ for some $n \geq 0$. Does it hold that $\alpha_{1}=\alpha_{2}=\alpha_{3} \cdots$ ?

\section{References}

[1] Y. Choi, A propagation of quadratically hyponormal weighted shifts, Bull. Korean Math. Soc., 37(2000), 347-352.

[2] R. Curto, Quadratically hyponormal weighted shifts, Integral Equations and Operator Theory, 13(1990), 49-66.

[3] R. Curto, Joint hyponormality: A bridge between hyponormality and subnormality, Proc. Symposia Pure Math., 51(1990), Part II, 69-91.

[4] R. Curto and L. Fialkow, Recursively generated weighted shifts and the subnormal completion problem, I, Integral Equations and Operator Theory, 17(1993), 202-246.

[5] R. Curto and L. Fialkow, Recursively generated weighted shifts and the subnormal completion problem, II, Integral Equations and Operator Theory, 18(1994), 369-426.

[6] R. Curto and I. Jung, Quadratically hyponormal weighted shifts with two equal weights, Integral Equations and Operator Theory, 37(2000), 208-231.

[7] R. Curto and M. Putinar, Nearly subnormal operators and moment problems, J. Funct. Anal., 115(1993), 480-497.

[8] I. Jung and S. Park, Quadratically hyponormal weighted shifts and their examples, Integral Equations and Operator Theory, 36(2000), 480-498.

[9] I. Jung and S. Park, Cubically hyponormal weighted shifts and their examples, J. Math. Anal. Appl., 247(2000), 557-569.

[10] J. Stampfli, Which weighted shifts are subnormal, Pacific J. Math., 17(1966), 367-379.

[11] R. Sweet, A recursive relation for the determinant of a pentadiagonal matrix, Comm ACM, 12(1969), 330-332.

[12] Wolfram Research, Inc. Mathematica, Version 3.0, Wolfram Research Inc., Champaign, IL, 1996. 